\title{
Soliton Solutions of the Klein-Gordon-Zakharov Equation with Power Law Nonlinearity
}

\author{
Mehmet Ekici, ${ }^{1}$ Durgun Duran, ${ }^{2}$ and Abdullah Sonmezoglu ${ }^{1}$ \\ ${ }^{1}$ Department of Mathematics, Faculty of Science and Arts, Bozok University, 66100 Yozgat, Turkey \\ ${ }^{2}$ Department of Physics, Faculty of Science and Arts, Bozok University, 66100 Yozgat, Turkey
}

Correspondence should be addressed to Mehmet Ekici; ekici-m@hotmail.com

Received 30 August 2013; Accepted 8 October 2013

Academic Editors: L. Pan, V. Rai, and B. Tadić

Copyright (C) 2013 Mehmet Ekici et al. This is an open access article distributed under the Creative Commons Attribution License, which permits unrestricted use, distribution, and reproduction in any medium, provided the original work is properly cited.

\begin{abstract}
We introduce a new version of the trial equation method for solving nonintegrable partial differential equations in mathematical physics. Some exact solutions including soliton solutions and rational and elliptic function solutions to the Klein-Gordon-Zakharov equation with power law nonlinearity in $(1+2)$ dimensions are obtained by this method.
\end{abstract}

\section{Introduction}

In recent years there have been many works on the qualitative research of the global solutions for the Klein-GordonZakharov (KGZ) equations [1-4]. Chen considered orbital stability of solitary waves for the KGZ equations in [5]. More recently, some exact solutions for the Zakharov equations are obtained by using different methods [6-9]. These solutions are not general and by no means exhaust all possibilities. They are only some particular solutions within some specific parameters choices.

The aim of this paper is to find the new and more general explicit and exact special solutions of the KGZ equations. We obtain various of explicit and exact special solutions of the KGZ equations by using the extended trial equation method. These solutions include that of the solitary wave solutions of the singular traveling wave solutions and solitary wave solutions of rational function type.

Solving nonlinear evolution equations has become a valuable task in many scientific areas including applied mathematics as well as the physical sciences and engineering. Many powerful methods, such as the Backlund transformation, the inverse scattering method [10], bilinear transformation, the tanh-sech method [11], the extended tanh method, the pseudospectral method [12], the trial function and the sinecosine method [13], Hirota method [14], tanh-coth method $[15,16]$, the exponential function method [17], $\left(G^{\prime} / G\right)$ expansion method $[18,19]$, homogeneous balance method
[20], and the trial equation method [21-30] have been used to investigate nonlinear partial differential equations problems. There are a lot of nonlinear evolution equations that are integrated using these and other mathematical methods.

In this paper, KGZ equations will be studied by extended trial equation. By virtue of the solitary wave ansatz method, an exact soliton solution will be obtained. The extended trial equation method will be employed to back up our analysis in obtaining exact solutions with distinct physical structures.

\section{The Extended Trial Equation Method}

The main steps of an extended trial equation method for the nonlinear partial differential equations with higher order nonlinearity are outlined as follows.

Step 1. For a given nonlinear partial differential equation with rank inhomogeneous

$$
P\left(u, u_{t}, u_{x}, u_{x x}, \ldots\right)=0,
$$

take the wave transformation

$$
u\left(x_{1}, \ldots, x_{N}, t\right)=u(\eta), \quad \eta=\gamma\left(\sum_{j=1}^{N} x_{j}-c t\right)
$$


where $\gamma \neq 0$ and $c \neq 0$. Substituting (2) into (1) yields a nonlinear ordinary differential equation

$$
N\left(u, u^{\prime}, u^{\prime \prime}, \ldots\right)=0 .
$$

Step 2. Take transformation and trial equation as follows:

$$
u=\sum_{i=0}^{\delta} \tau_{i} \Gamma^{i}
$$

in which

$$
\left(\Gamma^{\prime}\right)^{2}=\Lambda(\Gamma)=\frac{\Phi(\Gamma)}{\Psi(\Gamma)}=\frac{\xi_{\theta} \Gamma^{\theta}+\cdots+\xi_{1} \Gamma+\xi_{0}}{\zeta_{\epsilon} \Gamma^{\epsilon}+\cdots+\zeta_{1} \Gamma+\zeta_{0}},
$$

where $\tau_{i}(i=0, \ldots, \delta), \xi_{i}(i=0, \ldots, \theta)$, and $\zeta_{i}(i=0, \ldots, \epsilon)$ are constants. Using the relations (4) and (5), we can find

$$
\begin{gathered}
\left(u^{\prime}\right)^{2}=\frac{\Phi(\Gamma)}{\Psi(\Gamma)}\left(\sum_{i=0}^{\delta} i \tau_{i} \Gamma^{i-1}\right)^{2}, \\
u^{\prime \prime}=\frac{\Phi^{\prime}(\Gamma) \Psi(\Gamma)-\Phi(\Gamma) \Psi^{\prime}(\Gamma)}{2 \Psi^{2}(\Gamma)}\left(\sum_{i=0}^{\delta} i \tau_{i} \Gamma^{i-1}\right) \\
+\frac{\Phi(\Gamma)}{\Psi(\Gamma)}\left(\sum_{i=0}^{\delta} i(i-1) \tau_{i} \Gamma^{i-2}\right),
\end{gathered}
$$

where $\Phi(\Gamma)$ and $\Psi(\Gamma)$ are polynomials. Substituting these terms into (3) yields an equation of polynomial $\Omega(\Gamma)$ of $\Gamma$ as follows:

$$
\Omega(\Gamma)=\varrho_{s} \Gamma^{s}+\cdots+\varrho_{1} \Gamma+\varrho_{0}=0 .
$$

According to the balance principle we can determine a relation of $\theta, \epsilon$, and $\delta$. We can take some values of $\theta, \epsilon$, and $\delta$.

Step 3. Let the coefficients of $\Omega(\Gamma)$ all be zero, this will yield the following algebraic equations system:

$$
\varrho_{i}=0, \quad i=0, \ldots, s .
$$

Solving this equation system (8), we will determine the values of $\xi_{0}, \ldots, \xi_{\theta} ; \zeta_{0}, \ldots, \zeta_{\epsilon}$, and $\tau_{0}, \ldots, \tau_{\delta}$.

Step 4. Reduce (5) to the elementary integral form as follows:

$$
\pm\left(\eta-\eta_{0}\right)=\int \frac{d \Gamma}{\sqrt{\Lambda(\Gamma)}}=\int \sqrt{\frac{\Psi(\Gamma)}{\Phi(\Gamma)}} d \Gamma .
$$

Using a complete discrimination system for polynomial to classify the roots of $\Phi(\Gamma)$, we solve the infinite integral (9) and obtain the exact solutions to (3). Furthermore, we can write the exact traveling wave solutions to (1), respectively.

\section{Mathematical Analysis}

We introduce the KGZ equation with power law nonlinearity in $(1+2)$ dimensions and its soliton solution by extended trial equation method and show its numerical solution at a fixed point.
3.1. The KGZ Equation in (1 + 2) Dimensions. The dimensionless form of the KGZ equation in $(1+2)$ dimensions that will be studied in this subsection is given by [31]

$$
\begin{aligned}
& q_{t t}-\lambda^{2}\left(q_{x x}+q_{y y}\right)+q+r q+\alpha|q|^{2 m} q=0, \\
& r_{t t}-\lambda^{2}\left(r_{x x}+r_{y y}\right)=\left(|q|^{2 m}\right)_{x x}+\left(|q|^{2 m}\right)_{y y} .
\end{aligned}
$$

Here, the dependent variables are $q$ and $r$, while the independent variables are $x, y$, and $t$ which are, respectively, referred to as the spatial variables and temporal variable. Power law nonlinearity arises in nonlinear plasmas that solves the problem of small K-condensation in weak turbulence theory. It also arises in the context of nonlinear optics. The parameter $m>0$ dictates the power law nonlinearity, while $\lambda$ and $\alpha$ are constants. Here, in (10) and (11), $q$ is a complex valued function while $r$ is a real valued function. Equations (10) and (11) together appear in the area of Plasma Physics. They describe the interaction of Langmuir waves and ionacoustic waves in plasmas [32, 33]. For solving (10) and (11) with the trial equation method, using the wave variables

$$
\begin{gathered}
q(x, y, t)=u(\eta) e^{i \phi}, \quad r(x, y, t)=v(\eta) \\
\phi=-\kappa_{1} x-\kappa_{2} y+\omega t+\varsigma, \quad \eta=\beta_{1} x+\beta_{2} y-c t,
\end{gathered}
$$

where $\kappa_{1}, \kappa_{2}, \omega, \varsigma, \beta_{1}, \beta_{2}$, and $c$ are real constants, (10) and (11) are converted to the system of ODEs

$$
\begin{gathered}
\left(w c-\lambda^{2}\left(\kappa_{1} \beta_{1}+\kappa_{2} \beta_{2}\right)\right) u^{\prime}(\eta)=0 \\
\left(1-\omega^{2}+\lambda^{2}\left(\kappa_{1}^{2}+\kappa_{2}^{2}\right)\right) u(\eta) \\
+\left(c^{2}-\lambda^{2}\left(\beta_{1}^{2}+\beta_{2}^{2}\right)\right) u^{\prime \prime}(\eta) \\
+\alpha u^{2 m+1}(\eta)+u(\eta) v(\eta)=0, \\
\left(c^{2}-\lambda^{2}\left(\beta_{1}^{2}+\beta_{2}^{2}\right)\right) v^{\prime \prime}(\eta)-\left(\beta_{1}^{2}+\beta_{2}^{2}\right)\left(u^{2 m}\right)^{\prime \prime}(\eta)=0
\end{gathered}
$$

where primes denote the derivatives with respect to $\eta$. Equation (15) is then integrated term by term two times where integration constants are considered zero. This converts it into

$$
v(\eta)=\frac{\beta_{1}^{2}+\beta_{2}^{2}}{c^{2}-\lambda^{2}\left(\beta_{1}^{2}+\beta_{2}^{2}\right)} u^{2 m}(\eta) .
$$

Substituting (16) into (14) gives

$$
\begin{aligned}
(1- & \left.\omega^{2}+\lambda^{2}\left(\kappa_{1}^{2}+\kappa_{2}^{2}\right)\right) u(\eta) \\
& +\left(c^{2}-\lambda^{2}\left(\beta_{1}^{2}+\beta_{2}^{2}\right)\right) u^{\prime \prime}(\eta) \\
& +\left(\alpha+\frac{\beta_{1}^{2}+\beta_{2}^{2}}{c^{2}-\lambda^{2}\left(\beta_{1}^{2}+\beta_{2}^{2}\right)}\right) u^{2 m+1}(\eta)=0 .
\end{aligned}
$$

Equation (17), with the transformation

$$
u(\eta)=V^{1 / m}(\eta),
$$


reduces to

$$
\begin{gathered}
P\left(c^{2}-Q\right) V V^{\prime \prime}+\left(P^{2}-P\right)\left(c^{2}-Q\right)\left(V^{\prime}\right)^{2}+\left(R-w^{2}\right) V^{2} \\
+\left(\alpha+\frac{Q}{\lambda^{2}\left(c^{2}-Q\right)}\right) V^{4}=0,
\end{gathered}
$$

where

$$
\begin{gathered}
P=\frac{1}{m}, \quad Q=\lambda^{2}\left(\beta_{1}^{2}+\beta_{2}^{2}\right), \\
R=1+\lambda^{2}\left(\kappa_{1}^{2}+\kappa_{2}^{2}\right) .
\end{gathered}
$$

Substituting (6) into (19) and using balance principle yields $\theta=\epsilon+2 \delta+2$. If we take $\theta=4, \epsilon=0$, and $\delta=1$, then

$$
\left(V^{\prime}\right)^{2}=\frac{\tau_{1}^{2}\left(\xi_{4} \Gamma^{4}+\xi_{3} \Gamma^{3}+\xi_{2} \Gamma^{2}+\xi_{1} \Gamma+\xi_{0}\right)}{\zeta_{0}},
$$

where $\xi_{4} \neq 0$ and $\zeta_{0} \neq 0$. Solving the algebraic equation system (8) yields

$$
\begin{gathered}
\xi_{0}=\frac{-\zeta_{0} \tau_{0}^{4} P Q+\lambda^{2} \zeta_{0} \tau_{0}^{2}\left(Q-c^{2}\right)\left(\alpha \tau_{0}^{2} P+(1+P)\left(R-\omega^{2}\right)\right)}{\lambda^{2} \tau_{1}^{2} P^{2}(1+P)\left(c^{2}-Q\right)^{2}}, \\
\xi_{3}=-\frac{4 \zeta_{0} \tau_{0} \tau_{1}\left(Q+\alpha \lambda^{2}\left(c^{2}-Q\right)\right)}{\lambda^{2} P(1+P)\left(c^{2}-Q\right)^{2}}, \\
\xi_{1}=\frac{-4 \zeta_{0} \tau_{0}^{3} P Q+2 \lambda^{2} \zeta_{0} \tau_{0}\left(Q-c^{2}\right)\left(2 \alpha \tau_{0}^{2} P+(1+P)\left(R-\omega^{2}\right)\right)}{\lambda^{2} \tau_{1} P^{2}(1+P)\left(c^{2}-Q\right)^{2}}, \\
\xi_{4}=-\frac{\zeta_{0} \tau_{1}^{2}\left(Q+\alpha \lambda^{2}\left(c^{2}-Q\right)\right)}{\lambda^{2} P(1+P)\left(c^{2}-Q\right)^{2}}, \\
\xi_{2}=\frac{-6 \zeta_{0} \tau_{0}^{2} P Q+\lambda^{2} \zeta_{0}\left(Q-c^{2}\right)\left(6 \alpha \tau_{0}^{2} P+(1+P)\left(R-\omega^{2}\right)\right)}{\lambda^{2} P^{2}(1+P)\left(c^{2}-Q\right)^{2}} \\
\zeta_{0}=\zeta_{0}, \quad \tau_{0}=\tau_{0},
\end{gathered}
$$

Also from (13), it can be seen that $w c=\lambda^{2}\left(\kappa_{1} \beta_{1}+\kappa_{2} \beta_{2}\right)$. Substituting these results into (5) and (9), we can write

$$
\begin{aligned}
\pm\left(\eta-\eta_{0}\right)= & \sqrt{-\frac{\lambda^{2} P(1+P)\left(c^{2}-Q\right)^{2}}{\tau_{1}^{2}\left(Q+\alpha \lambda^{2}\left(c^{2}-Q\right)\right)}} \\
& \times \int \frac{d \Gamma}{\sqrt{\Gamma^{4}+\ell_{3} \Gamma^{3}+\ell_{2} \Gamma^{2}+\ell_{1} \Gamma+\ell_{0}}}
\end{aligned}
$$

where

$$
\begin{gathered}
\ell_{3}=\frac{4 \tau_{0}}{\tau_{1}} \\
\ell_{2}=\frac{-6 \tau_{0}^{2} P Q+\lambda^{2}\left(Q-c^{2}\right)\left(6 \alpha \tau_{0}^{2} P+(1+P)\left(R-\omega^{2}\right)\right)}{\tau_{1}^{2} P\left(-Q+\alpha \lambda^{2}\left(Q-c^{2}\right)\right)}, \\
\ell_{1}=\frac{-4 \tau_{0}^{3} P Q+2 \lambda^{2} \tau_{0}\left(Q-c^{2}\right)\left(2 \alpha \tau_{0}^{2} P+(1+P)\left(R-\omega^{2}\right)\right)}{\tau_{1}^{3} P\left(-Q+\alpha \lambda^{2}\left(Q-c^{2}\right)\right)}, \\
\ell_{0}=\frac{\zeta_{0} \tau_{0}^{4} P Q-\lambda^{2} \zeta_{0} \tau_{0}^{2}\left(Q-c^{2}\right)\left(\alpha \tau_{0}^{2} P+(1+P)\left(R-\omega^{2}\right)\right)}{\zeta_{0} \tau_{1}^{4} P\left(Q+\alpha \lambda^{2}\left(c^{2}-Q\right)\right)} .
\end{gathered}
$$

Integrating (23), we obtain the solutions to (10) and (11) as follows:

$$
\begin{gathered}
\pm\left(\eta-\eta_{0}\right)=-\frac{B}{\Gamma-\alpha_{1}}, \\
\pm\left(\eta-\eta_{0}\right)=\frac{2 B}{\alpha_{1}-\alpha_{2}} \sqrt{\frac{\Gamma-\alpha_{2}}{\Gamma-\alpha_{1}}}, \quad \alpha_{2}>\alpha_{1}, \\
\pm\left(\eta-\eta_{0}\right)=\frac{B}{\alpha_{1}-\alpha_{2}} \ln \left|\frac{\Gamma-\alpha_{1}}{\Gamma-\alpha_{2}}\right| \\
\pm\left(\eta-\eta_{0}\right)=\frac{B}{\sqrt{\left(\alpha_{1}-\alpha_{2}\right)\left(\alpha_{1}-\alpha_{3}\right)}} \\
\times \ln \left|\frac{\sqrt{\left(\Gamma-\alpha_{2}\right)\left(\alpha_{1}-\alpha_{3}\right)}-\sqrt{\left(\Gamma-\alpha_{3}\right)\left(\alpha_{1}-\alpha_{2}\right)}}{\sqrt{\left(\Gamma-\alpha_{2}\right)\left(\alpha_{1}-\alpha_{3}\right)}+\sqrt{\left(\Gamma-\alpha_{3}\right)\left(\alpha_{1}-\alpha_{2}\right)}}\right| \\
\pm\left(\eta-\eta_{0}\right)=2 \sqrt{\frac{B}{\left(\alpha_{1}-\alpha_{3}\right)\left(\alpha_{2}-\alpha_{4}\right)} F(\varphi, l),} \\
\alpha_{1}>\alpha_{2}>\alpha_{3}>\alpha_{4},
\end{gathered}
$$

where

$$
\begin{gathered}
B=\sqrt{-\frac{\lambda^{2} P(1+P)\left(c^{2}-Q\right)^{2}}{\tau_{1}^{2}\left(Q+\alpha \lambda^{2}\left(c^{2}-Q\right)\right)}}, \\
F(\varphi, l)=\int_{0}^{\varphi} \frac{d \psi}{\sqrt{1-l^{2} \sin ^{2} \psi}}, \\
\varphi=\arcsin \sqrt{\frac{\left(\Gamma-\alpha_{1}\right)\left(\alpha_{2}-\alpha_{4}\right)}{\left(\Gamma-\alpha_{2}\right)\left(\alpha_{1}-\alpha_{4}\right)}} \\
l^{2}=\frac{\left(\alpha_{2}-\alpha_{3}\right)\left(\alpha_{1}-\alpha_{4}\right)}{\left(\alpha_{1}-\alpha_{3}\right)\left(\alpha_{2}-\alpha_{4}\right)} .
\end{gathered}
$$


Also $\alpha_{1}, \alpha_{2}, \alpha_{3}$, and $\alpha_{4}$ are the roots of the polynomial equation

$$
\Gamma^{4}+\frac{\xi_{3}}{\xi_{4}} \Gamma^{3}+\frac{\xi_{2}}{\xi_{4}} \Gamma^{2}+\frac{\xi_{1}}{\xi_{4}} \Gamma+\frac{\xi_{0}}{\xi_{4}}=0
$$

Substituting the solutions (25)-(28) into (4) and (18), we obtain, respectively,

$$
\begin{aligned}
& q(x, y, t) \\
& =\left\{\tau_{0}+\tau_{1} \alpha_{1} \pm \frac{\tau_{1} B}{\beta_{1} x+\beta_{2} y-c t-\eta_{0}}\right\}^{1 / m} e^{i \phi}, \\
& r(x, y, t) \\
& =\frac{\beta_{1}^{2}+\beta_{2}^{2}}{c^{2}-\lambda^{2}\left(\beta_{1}^{2}+\beta_{2}^{2}\right)} \\
& \times\left\{\tau_{0}+\tau_{1} \alpha_{1} \pm \frac{\tau_{1} B}{\beta_{1} x+\beta_{2} y-c t-\eta_{0}}\right\}^{2}, \\
& q(x, y, t) \\
& =\left\{\tau_{0}+\tau_{1} \alpha_{1}\right. \\
& \left.+\frac{4 B^{2}\left(\alpha_{2}-\alpha_{1}\right) \tau_{1}}{4 B^{2}-\left[\left(\alpha_{1}-\alpha_{2}\right)\left(\beta_{1} x+\beta_{2} y-c t-\eta_{0}\right)\right]^{2}}\right\}^{1 / m} e^{i \phi}, \\
& r(x, y, t) \\
& =\frac{\beta_{1}^{2}+\beta_{2}^{2}}{c^{2}-\lambda^{2}\left(\beta_{1}^{2}+\beta_{2}^{2}\right)} \\
& \times\left\{\tau_{0}+\tau_{1} \alpha_{1}\right. \\
& \left.+\frac{4 B^{2}\left(\alpha_{2}-\alpha_{1}\right) \tau_{1}}{4 B^{2}-\left[\left(\alpha_{1}-\alpha_{2}\right)\left(\beta_{1} x+\beta_{2} y-c t-\eta_{0}\right)\right]^{2}}\right\}^{2}, \\
& q(x, y, t) \\
& =\left\{\tau_{0}+\tau_{1} \alpha_{2}+\left(\left(\alpha_{2}-\alpha_{1}\right) \tau_{1}\right)\right. \\
& \left.\times\left(\exp \left(\frac{\alpha_{1}-\alpha_{2}}{B}\left(\beta_{1} x+\beta_{2} y-c t-\eta_{0}\right)\right)-1\right)^{-1}\right\}^{1 / m} e^{i \phi},
\end{aligned}
$$

$$
r(x, y, t)
$$

$$
\begin{aligned}
& =\frac{\beta_{1}^{2}+\beta_{2}^{2}}{c^{2}-\lambda^{2}\left(\beta_{1}^{2}+\beta_{2}^{2}\right)} \\
& \quad \times\left\{\tau_{0}+\tau_{1} \alpha_{2}+\left(\left(\alpha_{2}-\alpha_{1}\right) \tau_{1}\right)\right. \\
& \left.\quad \times\left(\exp \left(\frac{\alpha_{1}-\alpha_{2}}{B}\left(\beta_{1} x+\beta_{2} y-c t-\eta_{0}\right)\right)-1\right)^{-1}\right\}^{2},
\end{aligned}
$$

$$
\begin{aligned}
& q(x, y, t) \\
& =\left\{\tau_{0}+\tau_{1} \alpha_{1}+\left(\left(\alpha_{1}-\alpha_{2}\right) \tau_{1}\right)\right. \\
& \left.\quad \times\left(\exp \left(\frac{\alpha_{1}-\alpha_{2}}{B}\left(\beta_{1} x+\beta_{2} y-c t-\eta_{0}\right)\right)-1\right)^{-1}\right\}^{1 / m} e^{i \phi},
\end{aligned}
$$

$$
\begin{aligned}
& r(x, y, t) \\
& =\frac{\beta_{1}^{2}+\beta_{2}^{2}}{c^{2}-\lambda^{2}\left(\beta_{1}^{2}+\beta_{2}^{2}\right)} \\
& \quad \times\left\{\tau_{0}+\tau_{1} \alpha_{1}+\left(\left(\alpha_{1}-\alpha_{2}\right) \tau_{1}\right)\right. \\
& \left.\quad \times\left(\exp \left(\frac{\alpha_{1}-\alpha_{2}}{B}\left(\beta_{1} x+\beta_{2} y-c t-\eta_{0}\right)\right)-1\right)^{-1}\right\}^{2},
\end{aligned}
$$

$$
\begin{aligned}
& q(x, y, t) \\
& \quad \times\left(\begin{array}{l}
2 \alpha_{1}-\alpha_{2}-\alpha_{3}+\left(\alpha_{3}-\alpha_{2}\right) \\
\tau_{0}+\tau_{1} \alpha_{1}-\left(2\left(\alpha_{1}-\alpha_{2}\right)\left(\alpha_{1}-\alpha_{3}\right) \tau_{1}\right) \\
\times \cosh \left(\frac{\sqrt{\left(\alpha_{1}-\alpha_{2}\right)\left(\alpha_{1}-\alpha_{3}\right)}}{B}\right. \\
\left.\times\left(\beta_{1} x+\beta_{2} y-c t\right)\right)
\end{array}\right\} e^{i \phi},
\end{aligned}
$$

$$
\begin{aligned}
& r(x, y, t) \\
& =\frac{\beta_{1}^{2}+\beta_{2}^{2}}{c^{2}-\lambda^{2}\left(\beta_{1}^{2}+\beta_{2}^{2}\right)} \\
& \times\left\{\tau_{0}+\tau_{1} \alpha_{1}-\left(2\left(\alpha_{1}-\alpha_{2}\right)\left(\alpha_{1}-\alpha_{3}\right) \tau_{1}\right)\right. \\
& \times\left(2 \alpha_{1}-\alpha_{2}-\alpha_{3}+\left(\alpha_{3}-\alpha_{2}\right)\right. \\
& \times \cosh \left(\frac{\sqrt{\left(\alpha_{1}-\alpha_{2}\right)\left(\alpha_{1}-\alpha_{3}\right)}}{B}\right. \\
& \left.\left.\left.\times\left(\beta_{1} x+\beta_{2} y-c t\right)\right)\right)^{-1}\right\}^{2} .
\end{aligned}
$$


If we take $\tau_{0}=-\tau_{1} \alpha_{1}$ and $\eta_{0}=0$, then the solutions (32)-(41) can reduce to rational function solutions

$$
\begin{aligned}
& q(x, y, t)=\left( \pm \frac{\tau_{1} B}{\beta_{1} x+\beta_{2} y-c t}\right)^{1 / m} e^{i\left(-\kappa_{1} x-\kappa_{2} y+\omega t+\varsigma\right)}, \\
& r(x, y, t)=\Upsilon\left(\frac{\tau_{1} B}{\beta_{1} x+\beta_{2} y-c t}\right)^{2}, \\
& q(x, y, t)=\left\{\frac{4 B^{2}\left(\alpha_{2}-\alpha_{1}\right) \tau_{1}}{4 B^{2}-\left[\left(\alpha_{1}-\alpha_{2}\right)\left(\beta_{1} x+\beta_{2} y-c t\right)\right]^{2}}\right\}^{1 / m} \\
& \times e^{i\left(-\kappa_{1} x-\kappa_{2} y+\omega t+\varsigma\right)}, \\
& r(x, y, t)= \Upsilon\left\{\frac{4 B^{2}\left(\alpha_{2}-\alpha_{1}\right) \tau_{1}}{4 B^{2}-\left[\left(\alpha_{1}-\alpha_{2}\right)\left(\beta_{1} x+\beta_{2} y-c t\right)\right]^{2}}\right\}^{2},
\end{aligned}
$$

traveling wave solutions

$$
\begin{aligned}
& q(x, y, t) \\
& =\left\{\frac{\left(\alpha_{2}-\alpha_{1}\right) \tau_{1}}{2}\left\{1 \mp \operatorname{coth}\left[\frac{\alpha_{1}-\alpha_{2}}{2 B}\left(\beta_{1} x+\beta_{2} y-c t\right)\right]\right\}\right\}^{1 / m} \\
& \quad \times e^{i\left(-\kappa_{1} x-\kappa_{2} y+\omega t+\varsigma\right)}, \\
& r(x, y, t) \\
& =\Upsilon\left\{\frac{\left(\alpha_{2}-\alpha_{1}\right) \tau_{1}}{2}\left\{1 \mp \operatorname{coth}\left[\frac{\alpha_{1}-\alpha_{2}}{2 B}\left(\beta_{1} x+\beta_{2} y-c t\right)\right]\right\}\right\}^{2},
\end{aligned}
$$

and soliton solutions

$$
\begin{aligned}
q(x, y, t)= & \frac{A_{1}}{\left(D+\cosh \left[B_{1}\left(\beta_{1} x+\beta_{2} y-c t\right)\right]\right)^{1 / m}} \\
& \times e^{i\left(-\kappa_{1} x-\kappa_{2} y+\omega t+\varsigma\right)}, \\
r(x, y, t)= & \Upsilon \frac{A_{2}}{\left(D+\cosh \left[B_{1}\left(\beta_{1} x+\beta_{2} y-c t\right)\right]\right)^{2}},
\end{aligned}
$$

where

$$
\begin{gathered}
\Upsilon=\frac{\beta_{1}^{2}+\beta_{2}^{2}}{c^{2}-\lambda^{2}\left(\beta_{1}^{2}+\beta_{2}^{2}\right)}, \\
A_{1}=\left(\frac{2\left(\alpha_{1}-\alpha_{2}\right)\left(\alpha_{1}-\alpha_{3}\right) \tau_{1}}{\alpha_{2}-\alpha_{3}}\right)^{1 / m}, \quad A_{2}=A_{1}^{2 m}, \\
B_{1}=\frac{\sqrt{\left(\alpha_{1}-\alpha_{2}\right)\left(\alpha_{1}-\alpha_{3}\right)}}{B}, \quad D=\frac{2 \alpha_{1}-\alpha_{2}-\alpha_{3}}{\alpha_{3}-\alpha_{2}} .
\end{gathered}
$$

Here, $A_{1}$ and $A_{2}$ are the amplitudes of the solitons, $\beta_{1}$ and $\beta_{2}$ are the inverse widths of the solitons in the $x$ - and $y$ directions, respectively, while $c$ is the velocity of the soliton.

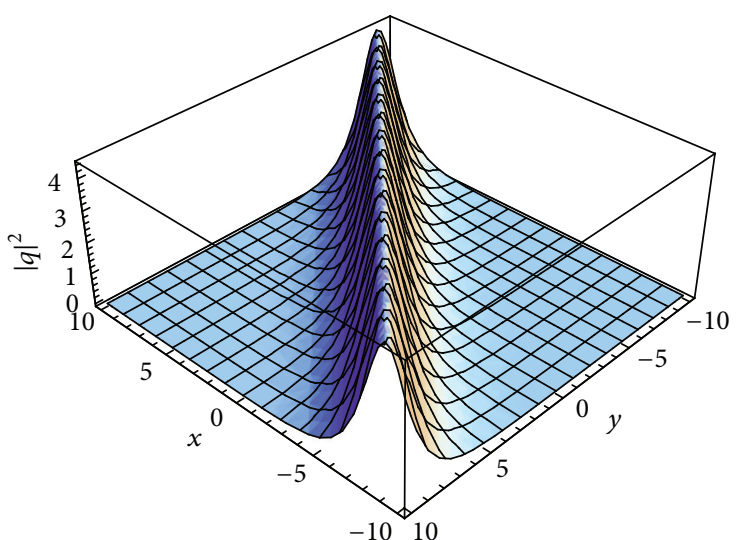

FIGURE 1: Profile of a numerical solution of (44) at $m=2, \beta_{1}=\beta_{2}=$ $1, A_{1}=2, B_{1}=0.8$, and $D<0$, while $c t=1$.

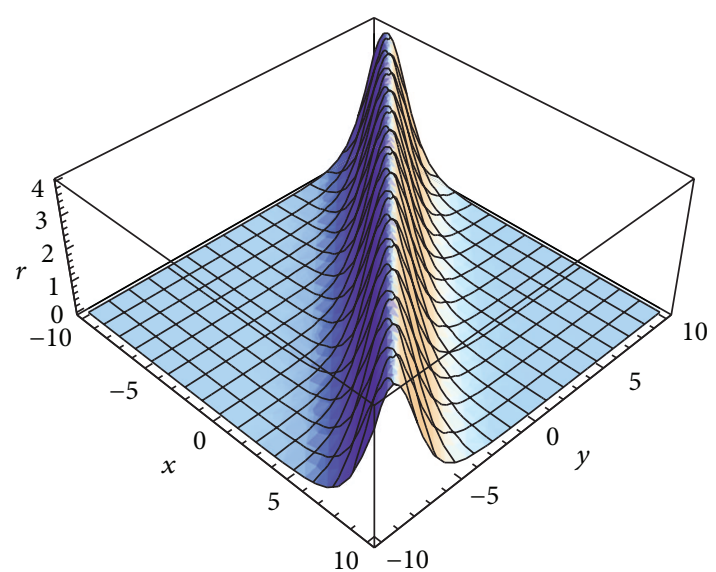

FIgURE 2: Profile of a numerical solution of (45) at $\beta_{1}=\beta_{2}=1$, $A_{2}=4, B_{1}=0.5, D<0$, and $c t=1$, while $\Upsilon>0$.

Also, $\kappa_{1}$ and $\kappa_{2}$ are the soliton frequencies in the $x$ - and $y$ directions, respectively, and $\omega$ is the soliton wave number, while $\varsigma$ is the phase constant. Thus, we can say that the solitons exist for $\tau_{1}>0$.

Remark. Ismail and Biswas obtained 1-soliton solution of KGZ equation in [31], we obtained soliton solution of this equation. In case $2 \alpha_{1}=\alpha_{2}+\alpha_{3}$, our soliton solutions in (44) and (45) reduce to 1-soliton solutions in [31].

In Figures 1, 2, and 3, we give profiles of numerical soliton solutions of (44) and (45) for various values of parameters.

\section{Conclusion}

We adopt the extended trial equation method to obtain soliton solutions of the KGZ equations in plasma physics. We obtain some more general solitary wave solutions of the KGZ equations. It not only produces the same solutions but also can pick up what we believe to be new solutions missed by other authors. The results indicate the KGZ equations admit soliton solutions with some arbitrary parameters. The type of exact solitary wave solution is different along with 


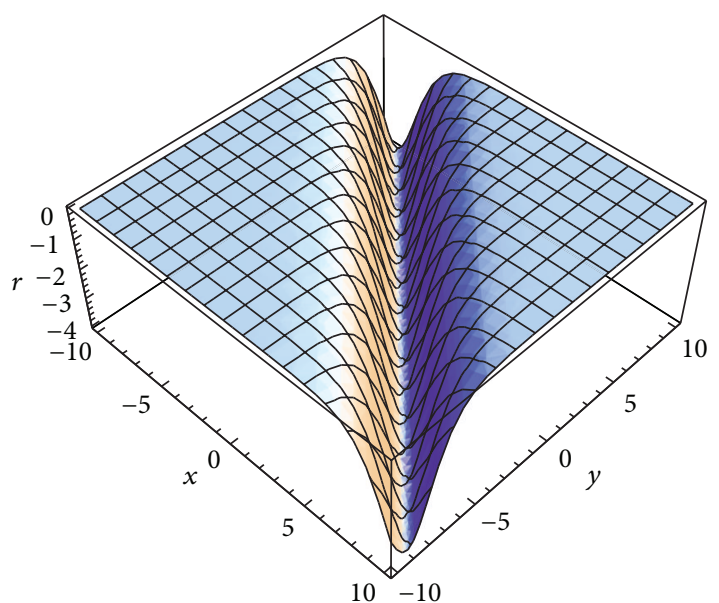

FIGURE 3: Profile of a numerical solution of (45) at $\beta_{1}=\beta_{2}=1$, $A_{2}=4, B_{1}=0.5, D<0$, and $c t=1$, while $\Upsilon<0$.

different value of arbitrary parameters. So we can choose appropriate parameter value to obtain solutions which we need in applications. The method can also be employed to solve a large number of other nonlinear evolution equations, such as nonlinear reaction-diffusion equation, the long-short wave resonance equation, the shallow water wave equation, Whitham-Broer-Kaup equation, variant Boussinesq equation, double Sine-Gardon equation, and Dodd-BulloughMikhailov equation.

\section{References}

[1] B. L. Guo and G. W. Yuan, "Global smooth solution for the Klein-Gordon-Zakharov equations," Journal of Mathematical Physics, vol. 36, no. 8, pp. 4119-4124, 1995.

[2] T. Ozawa, K. Tsutaya, and Y. Tsutsumi, "Normal form and global solutions for the Klein-Gordon-Zakharov equations," Annales de l'Institut Henri Poincaré Analyse Non Linéaire, vol. 12, no. 4, pp. 459-503, 1995.

[3] K. Tsutaya, "Global existence of small amplitude solutions for the Klein-Gordon-Zakharov equations," Nonlinear Analysis, Theory, Methods and Applications, vol. 27, no. 12, pp. 1373-1380, 1996.

[4] G. Adomian, "Non-perturbative solution of the Klein-GordonZakharov equation," Applied Mathematics and Computation, vol. 81, no. 1, pp. 89-92, 1997.

[5] L. Chen, "Orbital stability of solitary waves for the KleinGordon-Zakharov equations," Acta Mathematicae Applicatae Sinica, vol. 15, no. 1, pp. 54-64, 1999.

[6] J. L. Zhang, M. L. Wang, D. M. Chen, and Z. D. Fang, "The periodic wave solutions for two nonlinear evolution equations," Communications in Theoretical Physics, vol. 40, no. 2, pp. 129132, 2003.

[7] D. J. Huang and H. Q. Zhang, "Extended hyperbolic function method and new exact solitary wave solutions of Zakharov equations," Acta Physica Sinica, vol. 53, no. 8, pp. 2434-2438, 2004.
[8] Y. Chen and B. Li, "New exact travelling wave solutions for generalized Zakharov-Kuzentsov equations using general projective riccati equation method," Communications in Theoretical Physics, vol. 41, no. 1, pp. 1-6, 2004.

[9] Y. Chen, B. Li, and H.-Q. Zhang, "Bäcklund transformation and exact solutions for a new generalized Zakharov-Kuzentsov equation with nonlinear terms of any order," Communications in Theoretical Physics, vol. 39, no. 2, pp. 135-140, 2003.

[10] M. J. Ablowitz, P. A. Clarkson, and Solitons, Nonlinear Evolution Equations and Inverse Scattering, Cambridge University press, Cambridge, UK, 1991.

[11] A.-M. Wazwaz, "The tanh method for traveling wave solutions of nonlinear equations," Applied Mathematics and Computation, vol. 154, no. 3, pp. 713-723, 2004.

[12] P. Rosenau and J. M. Hyman, "Compactons: solitons with finite wavelength," Physical Review Letters, vol. 70, no. 5, pp. 564-567, 1993.

[13] A.-M. Wazwaz, "An analytic study of compactons structures in a class of nonlinear dispersive equations," Mathematics and Computers in Simulation, vol. 63, no. 1, pp. 35-44, 2003.

[14] R. Hirota, "Exact solution of the korteweg-de vries equation for multiple Collisions of solitons," Physical Review Letters, vol. 27, no. 18, pp. 1192-1194, 1971.

[15] W. Malfliet and W. Hereman, "The tanh method: I. Exact solutions of nonlinear evolution and wave equations," Physica Scripta, vol. 54, no. 6, pp. 563-568, 1996.

[16] M. A. Abdou, "The extended tanh method and its applications for solving nonlinear physical models," Applied Mathematics and Computation, vol. 190, no. 1, pp. 988-996, 2007.

[17] J.-H. He and X.-H. Wu, "Exp-function method for nonlinear wave equations," Chaos, Solitons and Fractals, vol. 30, no. 3, pp. 700-708, 2006.

[18] M. Wang, X. Li, and J. Zhang, “The $\left(G^{\prime} / G\right)$-expansion method and travelling wave solutions of nonlinear evolution equations in mathematical physics," Physics Letters A, vol. 372, no. 4, pp. 417-423, 2008.

[19] G. Ebadi and A. Biswas, "The $\left(G^{\prime} / G\right)$ method and topological soliton solution of the $\mathrm{K}(\mathrm{m}, \mathrm{n})$ equation," Communications in Nonlinear Science and Numerical Simulation, vol. 16, no. 6, pp. 2377-2382, 2011.

[20] M. Wang, "Solitary wave solutions for variant Boussinesq equations," Physics Letters A, vol. 199, no. 3-4, pp. 169-172, 1995.

[21] C.-S. Liu, "Trial equation method and its applications to nonlinear evolution equations," Acta Physica Sinica, vol. 54, no. 6, pp. 2505-2509, 2005.

[22] C.-S. Liu, "A new trial equation method and its applications," Communications in Theoretical Physics, vol. 45, no. 3, pp. 395397, 2006.

[23] C.-S. Liu, “Trial equation method to nonlinear evolution equations with rank inhomogeneous: mathematical discussions and its applications," Communications in Theoretical Physics, vol. 45, no. 2, pp. 219-223, 2006.

[24] C. S. Liu, "Using trial equation method to solve the exact solutions for two kinds of KdV equations with variable coefficients," Acta Physica Sinica, vol. 54, no. 10, pp. 4506-4510, 2005.

[25] C.-S. Liu, "Applications of complete discrimination system for polynomial for classifications of traveling wave solutions to nonlinear differential equations," Computer Physics Communications, vol. 181, no. 2, pp. 317-324, 2010.

[26] Y. Gurefe, A. Sonmezoglu, and E. Misirli, "Application of the trial equation method for solving some nonlinear evolution 
equations arising in mathematical physics," Pramana, vol. 77, no. 6, pp. 1023-1029, 2011.

[27] Y. Gurefe, A. Sonmezoglu, and E. Misirli, "Application of an irrational trial equation method to high-dimensional nonlinear evolution equations," Journal of Advanced Mathematical Studies, vol. 5, no. 1, pp. 41-47, 2012.

[28] Y. Pandir, Y. Gurefe, U. Kadak, and E. Misirli, "Classifications of exact solutions for some nonlinear partial differential equations with generalized evolution," Abstract and Applied Analysis, vol. 2012, Article ID 478531, 16 pages, 2012.

[29] Y. Gurefe, E. Misirli, A. Sonmezoglu, and M. Ekici, "Extended trial equation method to generalized nonlinear partial differential equations," Applied Mathematics and Computation, vol. 219, no. 10, pp. 5253-5260, 2013.

[30] Y. Gurefe, E. Misirli, Y. Pandir, A. Sonmezoglu, and M. Ekici, "New exact solutions of thedavey-stewartson equation with power-law nonlinearity," Bulletin of the Malaysian Mathematical Sciences Society. In press.

[31] M. S. Ismail and A. Biswas, "1-Soliton solution of the KleinGordon-Zakharov equation with power law nonlinearity," Applied Mathematics and Computation, vol. 217, no. 8, pp. 41864196, 2010.

[32] J. Wang, "Solitary wave propagation and interactions for the Klein-Gordon-Zakharov equations in plasma physics," Journal of Physics A, vol. 42, no. 8, pp. 085205-085221, 2009.

[33] T. Wang, J. Chen, and L. Zhang, "Conservative difference methods for the Klein-Gordon-Zakharov equations," Journal of Computational and Applied Mathematics, vol. 205, no. 1, pp. 430-452, 2007. 


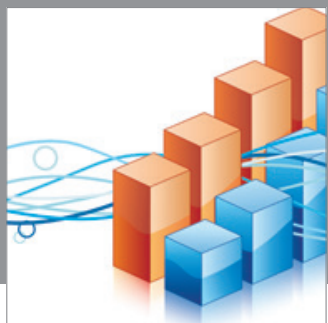

Advances in

Operations Research

mansans

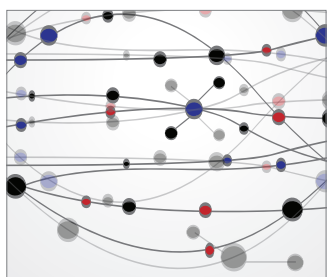

The Scientific World Journal
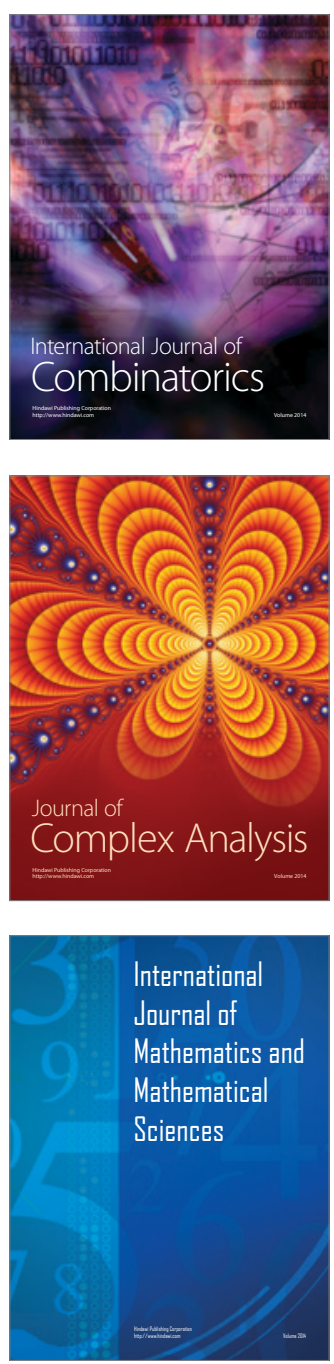
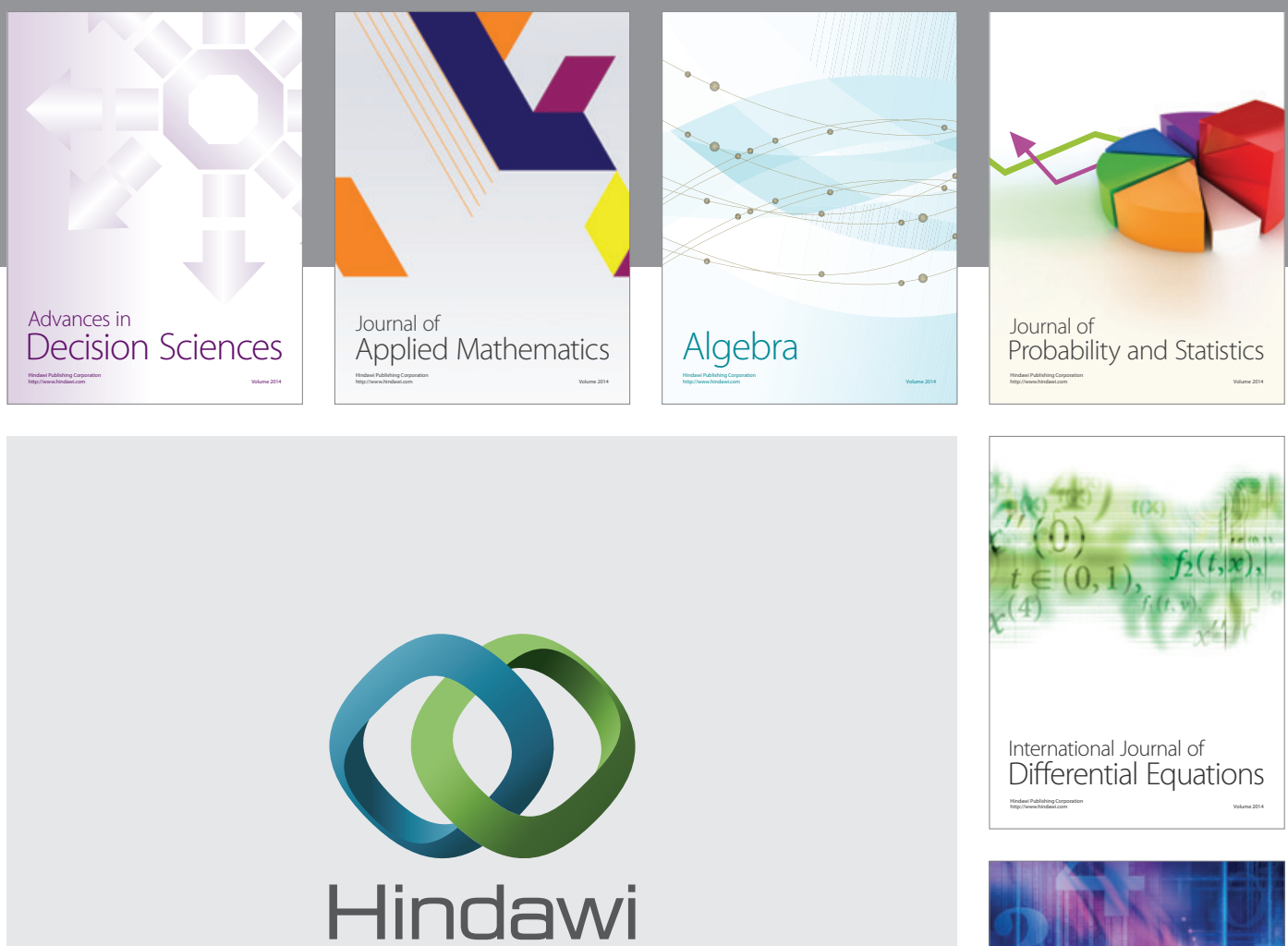

Submit your manuscripts at http://www.hindawi.com
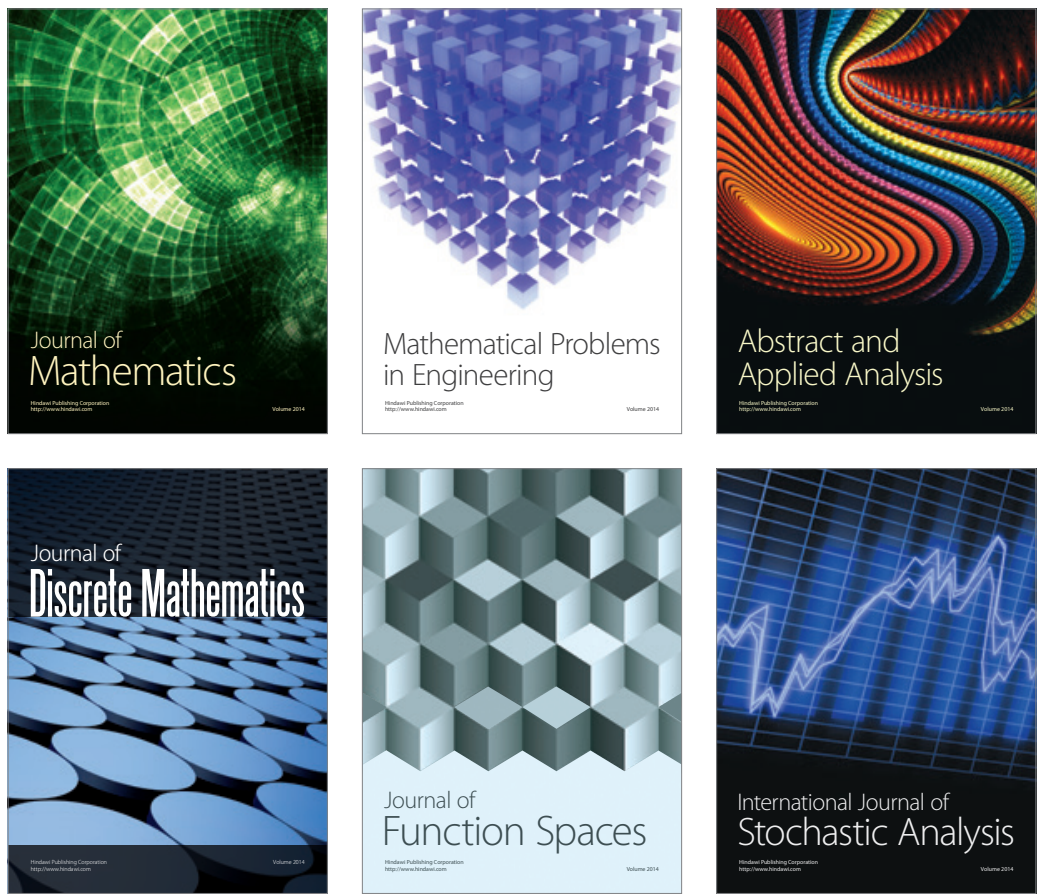

Journal of

Function Spaces

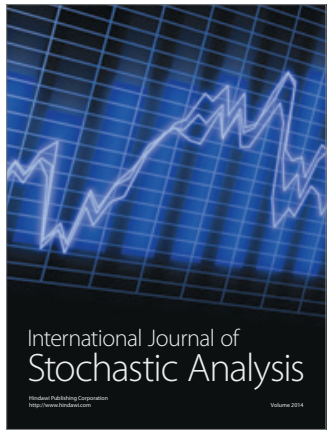

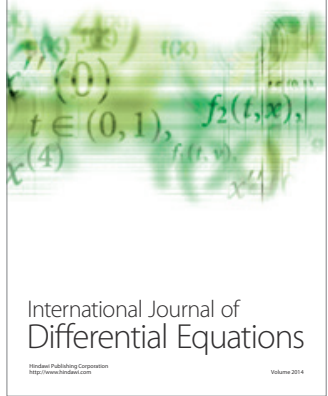
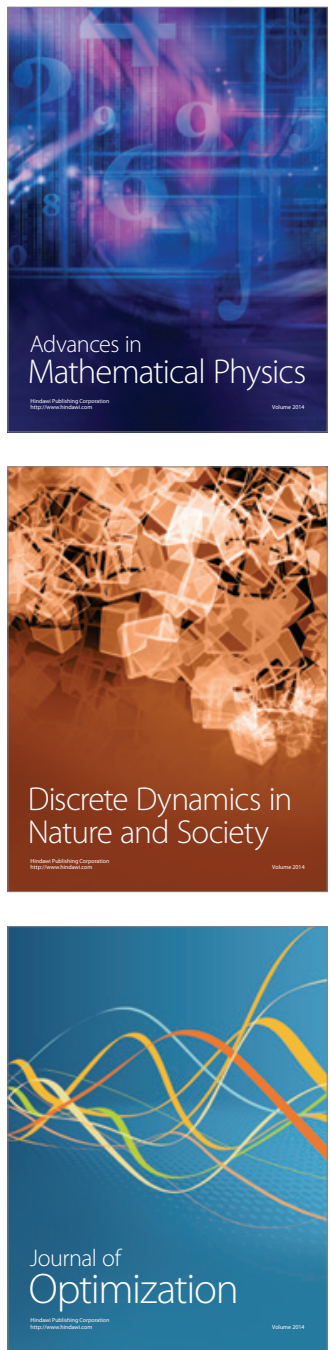